\title{
BEST APPROXIMATION IN CERTAIN DOUGLAS ALGEBRAS
}

\author{
RAHMAN YOUNIS
}

\begin{abstract}
The main result of this paper is that if $f \in L^{\infty}$ and $S$ is a weak peak set for $H^{\infty}$, then the distance from $f$ to the Douglas algebra $\left\{f \in L^{\infty}:\left.\left.f\right|_{s} \in H^{\infty}\right|_{s}\right\}$ is attained.
\end{abstract}

1. Introduction. Let $L^{\infty}$ denote the usual Lebesgue space of functions on the unit circle. Let $H^{\infty}$ denote the subalgebra of boundary values of bounded analytic functions on $|z|<1$. Let $H^{\infty}+C$ denote the closed linear span of $H^{\infty}$ and $C$, where $C$ is the set of continuous complex-valued functions on $|z|=1$.

The authors of [3] prove the following theorem.

Theorem A. If $f \in L^{\infty}$, then $\operatorname{dist}\left(f, H^{\infty}+C\right)=\|f-g\|_{\infty}$ for some $g$ in $H^{\infty}+$ C.

In [14], D. Luecking proves the following theorem.

TheOREM B. $\left(H^{\infty}+C\right) / H^{\infty}$ is an $M$-ideal in $L^{\infty} / H^{\infty}$.

Theorem B implies Theorem A. The author of [14] asked whether in Theorem B, $H^{\infty}+C$ can be replaced by other subalgebras. Also, D. Sarason [15, p. 113] and the authors of $\left[3\right.$, p. 609] asked whether in Theorem A, $H^{\infty}+C$ can be replaced by an arbitrary Douglas algebra.

In this paper, we prove a theorem (Theorem 3.1) which answers the question raised in [14], and its Corollary 3.2 gives a partial solution to the question raised in [3] and [15]. Theorem 4.1 is related to the general $L^{\infty}$ distance problem.

2. Preliminaries. We identify $L^{\infty}$ with $C(X)$, via the Gelfand transform, where $X$ is the maximal ideal space of $L^{\infty}$. Thus $H^{\infty}$ can be considered as a function algebra on $X$. No notational distinction will be made between $f$ in $L^{\infty}$ viewed as a function on the unit circle and its Gelfand transform $\hat{f}$ viewed as a continuous function on $X$.

A subset $S$ of $X$ is called a peak set for $H^{\infty}$ if there exists a function $f$ in $H^{\infty}$ such that $f=1$ on $S$ and $|f|<1$ off $S$. We say that $E$ is a weak peak set for $H^{\infty}$ if $E$ is the intersection of some collection of peak sets of $H^{\infty}$. If $S$ is a weak peak set for $H^{\infty}$, then $\left.H^{\infty}\right|_{S}$ is closed in $\left.L^{\infty}\right|_{S}$ [4, p. 104]. A subset $E$ of $X$ is called an antisymmetric set for a closed subalgebra $B\left(H^{\infty} \subset B \subset L^{\infty}\right)$ if $f \in B ; f$ is real on $E$ implies that $f$ is constant on $E$. A maximal antisymmetric set for $B$ is a closed

Received by the editors November 28, 1979.

1980 Mathematics Subject Classification. Primary 30H05, 46E15; Secondary 41A50.

Key words and phrases. $M$-ideal, weak peak set, best approximation. 
antisymmetric set which is not contained properly in any closed antisymmetric set for $B$. The reader is referred to [12] for many of the properties of $H^{\infty}$ and $L^{\infty}$ and to [4] and [9] for additional properties of function algebras.

Definition. A subspace $J$ of a Banach space $Y$ is called an $L$-ideal if there is a projection $E$ of $Y$ onto $J$ such that $\|y\|=\|E y\|+\|y-E y\|, y \in Y$. Such an $E$ is called an $L$-projection.

A subspace $K$ of a Banach space $X$ is called an $M$-ideal if the annihilator $K^{\perp}$ is an $L$-ideal of $X^{*}$ (see [1] for these concepts).

The following properties of an $M$-ideal of $X$ are needed in this paper.

Lemma C [1, Corollary 5.6]. If $K$ is an $M$-ideal of $X$ and if $x \in X$, then there exists $m \in K$ such that $\operatorname{dist}(x, K)=\|x-m\|$.

Lemma D [13, Theorem 3]. If $K$ is an $M$-ideal of $X$ and if $x \in X$, then the span of $P_{K}(x)=K$ if $x \notin K$, where $P_{K}(x)=\{m \in K$ : $\operatorname{dist}(x, K)=\|x-m\|\}$.

3. The main results. Let $S$ be a subset of $X$, the maximal ideal space of $L^{\infty}$, which is a weak peak set for $H^{\infty}$. Define $H_{S}^{\infty}=\left\{f \in L^{\infty}:\left.\left.f\right|_{S} \in H^{\infty}\right|_{s}\right\}$. It is a closed subalgebra of $L^{\infty}$ which contains $H^{\infty}$.

TheOREM 3.1. $H_{S}^{\infty} / H^{\infty}$ is an $M$-ideal in $L^{\infty} / H^{\infty}$.

Corollary 3.2. If $f \in L^{\infty}$, then $\operatorname{dist}\left(f, H_{S}^{\infty}\right)=\|f-h\|_{\infty}$ for some $h$ in $H_{S}^{\infty}$.

Proof of Corollary 3.2. Theorem 3.1 together with Lemma C imply that for any $f$ in $L^{\infty}$, there exists $g$ in $H_{S}^{\infty}$ such that $\operatorname{dist}\left(f, H_{S}^{\infty}\right)=\operatorname{dist}\left(f-g, H^{\infty}\right)$. Since $H^{\infty}$ is weak star closed in $L^{\infty}$, a compactness argument yields $g_{0}$ in $H^{\infty}$ such that $\operatorname{dist}\left(f-g, H^{\infty}\right)=\left\|f-g-g_{0}\right\|_{\infty}$. Set $h=g+g_{0}$; then $\operatorname{dist}\left(f, H_{S}^{\infty}\right)=\|f-h\|_{\infty}$.

Corollary 3.3. If $S \neq X$ and $f \notin H_{S}^{\infty}$ then the best approximation $h$ in Corollary 3.2 is not unique.

Indeed, if $f \notin H_{S}^{\infty}$, then by Lemma $\mathrm{D}, h$ is never unique.

Proof of Theorem 3.1. We identify $L^{\infty}$ with $C(X),\left(L^{\infty} / H^{\infty}\right)^{*}$ with

$$
\begin{aligned}
\left(H^{\infty}\right)^{\perp} & =\left\{\mu \in C(X)^{*}: \int f d \mu=0, f \in H^{\infty}\right\} \text { and } \\
\left(H_{S}^{\infty} / H^{\infty}\right)^{\perp} & =\left\{\mu \in C(X)^{*}: \int f d \mu=0, f \in H_{S}^{\infty}\right\} .
\end{aligned}
$$

To prove the theorem, we have to produce an $L$-projection $E$ of $\left(H^{\infty}\right)^{\perp}$ onto $\left(H_{s}^{\infty}\right)^{\perp}$.

Let $\mu \perp H^{\infty}$. Define $E_{\mu}=\chi_{s} \mu$, where $\chi_{s}$ denotes the characteristic function of $S$. By [4, p. 106], $\chi_{s} \mu \perp H^{\infty}$. It is easy to see that $H_{s}^{\infty}=H^{\infty}+J_{s}$, where $J_{s}=\left\{f \in L^{\infty}: f(S)=0\right\}$. In order to show that $\chi_{s} \mu \perp H_{s}^{\infty}$, it suffices to show that $\chi_{s} \mu \perp J_{s}$. But for $f$ in $J_{s}$, $\int f \chi_{s} d \mu=0$. Thus $E_{\mu} \perp H_{s}^{\infty}$. Note that $E^{2} \mu=E\left(\chi_{s} \mu\right)=\chi_{s}^{2} \mu=\chi_{s} \mu=E \mu$. Moreover, $\|\mu\|=\left\|\chi_{s} \mu+\left(1-\chi_{s}\right) \mu\right\|=\|E \mu\|$ $+\|\mu-E \mu\|$.

Finally, we have to show that $E$ is onto. Let $\mu \perp H_{s}^{\infty}$. Then $\mu \perp H^{\infty}+\mu \perp J_{s}$. We claim that the support of $\mu(=\operatorname{supp} \mu)$ is contained in $S$. To see that, suppose 
there exists $x \in X \backslash S$ such that $x \in \operatorname{supp} \mu$. Let $W$ be a clopen set of $X$ such that $x \in W$ and $W \cap S=\varnothing$. Now

$$
\int \chi_{W} d|\mu|=\sup \left\{\left|\int_{\chi} g d \mu\right|: g \in L^{\infty}, 0<|g|<\chi_{W}\right\} .
$$

(See [11, p. 364].) Since $\chi_{W}=0$ on $S$, we have $g=0$ on $S$. Consequently, $|\mu|(W)=\int \chi_{W} d|\mu|=0$. This contradiction shows that supp $\mu$ lies in $S$. Hence $\left(1-\chi_{s}\right) \mu \equiv 0$. That is $\mu=\chi_{s} \mu$. Thus $E_{\mu}=\chi_{s} \mu=\mu$. This ends the proof of the theorem.

4. The general distance problem. Let $B$ be an arbitrary closed subalgebra of $L^{\infty}$ which contains $H^{\infty}$. If $S$ is a maximal antisymmetric set for $B$ then $\left.H^{\infty}\right|_{S}=\left.B\right|_{S}$ [2, p. 20]. Since $S$ is a weak peak set for $B$, by $\left[4\right.$, p. 104] $\left.B\right|_{s}$ is closed in $\left.L^{\infty}\right|_{s}$. Since $\left.H^{\infty}\right|_{S}$ is closed in $\left.L^{\infty}\right|_{S}$, this forces $S$ to be a weak peak set for $H^{\infty}[9$, p. 65].

TheORem 4.1. Let $B$ be a closed subalgebra of $L^{\infty}$ and $f$ be in $L^{\infty}$. Then for every maximal antisymmetric set $S$ for $B$, there exists $h$ in $H_{S}^{\infty}$ such that $\operatorname{dist}(f, B)=$ $\|f-h\|_{\infty}$.

We need the following lemma.

LEMMA E [7]. Let $u \in L^{\infty}$ and $S$ be a weak peak set for $H^{\infty}$. Then $\operatorname{dist}\left(u, H_{S}^{\infty}\right)=$ $\operatorname{dist}_{s}\left(u, H^{\infty}\right)$, where $\operatorname{dist}_{s}\left(u, H^{\infty}\right)=\inf \left\{\|u-h\|_{s}: h \in H^{\infty}\right\}$.

Proof of Theorem 4.1. Using [10, p. 419], there exists a maximal antisymmetric set $E$ of $B$ such that $\operatorname{dist}(f, B)=\operatorname{dist}\left(\left.f\right|_{E},\left.B\right|_{E}\right)$. Thus

$$
\operatorname{dist}(f, B)=\operatorname{dist}\left(\left.f\right|_{E},\left.B\right|_{E}\right)=\operatorname{dist}\left(\left.f\right|_{E},\left.H^{\infty}\right|_{E}\right)=\operatorname{dist}_{E}\left(f, H^{\infty}\right) .
$$

Hence by Lemma $\mathrm{E}$, we have $\operatorname{dist}(f, B)=\operatorname{dist}\left(f, H_{E}^{\infty}\right)$.

Let $S$ be an arbitrary maximal antisymmetric set for $B$. Then $B \subset H_{S}^{\infty} \cap H_{E}^{\infty} \subset$ $H_{E}^{\infty}$. Since $\operatorname{dist}(f, B)=\operatorname{dist}\left(f, H_{E}^{\infty}\right)$, we see that $\operatorname{dist}(f, B)=\operatorname{dist}\left(f, H_{S}^{\infty} \cap H_{E}^{\infty}\right)$. We claim that $H_{S}^{\infty} \cap H_{E}^{\infty}=H_{S \cup E}^{\infty}$. Assume the claim for a moment; then $\operatorname{dist}\left(f, H_{S \cup E}^{\infty}\right)=\operatorname{dist}(f, B)$. By Theorem 3.1, there exists $h \in H_{S \cup E}^{\infty}$ such that $\operatorname{dist}(f, B)=\|f-h\|_{\infty}$.

Now to prove the claim we note that $H_{S}^{\infty} \cap H_{E}^{\infty}$ contains $H_{S \cup E}^{\infty}$. To prove the reverse, it suffices, according to the Chang-Marshall theorem [6], to prove that any inner function which is invertible in $H_{S}^{\infty} \cap H_{E}^{\infty}$ is also invertible in $H_{S \cup E}^{\infty}$. Let $b$ be an inner function such that $\left.\left.\bar{b}\right|_{S} \in H^{\infty}\right|_{S}$ and $\left.\left.\bar{b}\right|_{E} \in H^{\infty}\right|_{E}$. Then $b(S)=a$ and $b(E)=c$, where $a$ and $c$ are constant numbers such that $|a|=1$ and $|c|=1$. Let $Q$ be a finite Blaschke product such that $Q(a)=\bar{a}$ and $Q(c)=\bar{c}$ (see [5], [16]). Then $\left.(Q \circ b)\right|_{S \cup E}=\left.\bar{b}\right|_{S \cup E}$. This shows that $\bar{b} \in H_{S \cup E}^{\infty}$. This proves the claim, and consequently this ends the proof of Theorem 4.1.

Remarks. (1) The proof of Theorem 4.1 shows that if $S_{1}, \ldots, S_{N}$ are maximal antisymmetric sets for $B$, then there exists $h$ in $\cap_{i=1}^{N} H_{S_{i}}^{\infty} \operatorname{such}$ that $\operatorname{dist}(f, B)=$ $\|f-h\|_{\infty}$. The author does not know if $h$ can be chosen to be in $\cap_{S} H_{S}^{\infty}=B$, where $S$ runs over all maximal antisymmetric sets for $B$. 
(2) Perhaps, one might think that Theorem 3.1 implies Theorem A. The answer is no. In fact, there does not exist a weak peak set $S$ for $H^{\infty}$ such that $H^{\infty}+C=$ $H_{S}^{\infty}$. To see that, assume that $H^{\infty}+C=H_{S}^{\infty}$ for some $S$. Let $W$ be a clopen set in $X$ which contains $S$. Then $\chi_{W} \in H_{S}^{\infty}$. Thus $\chi_{W} \in H^{\infty}+C$. This is impossible because the maximal ideal space of $H^{\infty}+C$ is connected (see [8, Corollary 6.42] and [12, p. 188]).

Acknowledgements. I would like to thank D. Patil and P. Ahern for helpful discussions.

\section{REFERENCES}

1. E. Alfsen and E. Effros, Structure in real Banach spaces, Ann. of Math. (2) 96 (1972), 98-173.

2. S. Axler, Subalgebras of $L^{\infty}$, Thesis, University of California at Berkeley, 1975.

3. S. Axler, I. D. Berg, N. Jewell and A. Shields, Approximation by compact operators and the space $H^{\infty}+C$, Ann. of Math. (2) 109 (1979), 601-612.

4. A. Browder, Introduction to function algebras, Benjamin, New York, 1969.

5. D. G. Cantor and R. R. Phelps, An elementary interpolation theorem, Proc. Amer. Math. Soc. 16 (1965), 523-525.

6. S. Y. Chang and D. E. Marshall, Some algebras of bounded analytic functions, Lecture Notes in Math., vol. 604, Springer-Verlag, New York, 1977, pp. 12-20.

7. K. Clancey and J. Gosselin, On the local theory of local Toeplitz operators, Illinois J. Math. 22 (1978), 449-458.

8. R. G. Douglas, Banach algebra techniques in operator theory, Academic Press, New York, 1972.

9. T. Gamelin, Uniform algebras, Prentice-Hall, Englewood Cliffs, N.J., 1969.

10. I. Glicksberg, Measures orthogonal to algebras and sets of antisymmetry, Trans. Amer. Math. Soc. 105 (1962), 415-435.

11. E. Hewitt and K. Stromberg, Real and abstract analysis, Springer-Verlag, New York, 1975.

12. K. Hoffman, Banach spaces of analytic functions, Prentice-Hall Studies in Modern Analysis, Prentice-Hall, Englewood Cliffs, N.J., 1962.

13. R. Holmes, B. Scrantan and J. Ward, Approximation from the space of compact operators and other M-ideals, Duke Math. J. 42 (1975), 259-269.

14. D. H. Luecking, The compact Hankel operators form an $M$-ideal in the space of Hankel operators, Proc. Amer. Math. Soc. 79 (1980), 222-224.

15. D. Sarason, Lecture notes on function theory of the unit circle, Virginia Polytechnic and State University, Blacksburg, Virginia, June 19-23, 1978.

16. R. Younis, Interpolation by a finite Blaschke product, Proc. Amer. Math. Soc. 78 (1980), 451-452.

Department of Mathematics, University OF Wisconsin, MinwaUkBe, Wisconsin 53201 